\title{
Ising thin films with modulations and surface defects
}

\author{
W. Selke ${ }^{\mathrm{a}, 1}$, M. Pleimling ${ }^{\mathrm{b}, \mathrm{c}}$, I. Peschel $^{\mathrm{d}}$, M. Kaulke $^{\mathrm{d}}$, \\ M.-C. Chung d and D. Catrein ${ }^{\mathrm{a}}$ \\ ${ }^{a}$ Institut für Theoretische Physik, Technische Hochschule, D-52056 Aachen, \\ Germany \\ b Institut für Theoretische Physik 1, Universität Erlangen-Nürnberg, D-91058 \\ Erlangen, Germany \\ ${ }^{\mathrm{c}}$ Laboratoire de Physique des Matériaux, Université Henri Poincaré Nancy I, B.P. \\ 239, F-54506 Vandouvre lès Nancy Cedex, France \\ ${ }^{\mathrm{d}}$ Fachbereich Physik, Freie Universität Berlin, D-14195 Berlin, Germany
}

\begin{abstract}
Properties of magnetic films are studied in the framework of Ising models. In particular, we discuss critical phenomena of ferromagnetic Ising films with straight lines of magnetic adatoms and straight steps on the surface as well as phase diagrams of the axial next-nearest neighbour Ising (ANNNI) model for thin films exhibiting various spatially modulated phases.
\end{abstract}

Key words: Ising model, thin films, competing interactions, surface

1 Corresponding author: tel: +49-241-807029; fax: +49-241-8888188; e-mail: selke@physik.rwth-aachen.de

Preprint submitted to Elsevier Preprint 8 November 2018 
Magnetism in thin films has attracted much interest, both experimentally and theoretically $[1,2]$. In this paper, we shall deal, in the framework of Ising models, with critical properties of films having different types of surface defects as well as with phase diagrams of thin films displaying spatially modulated magnetic structures, extending our recent work $[3,4]$.

To analyse the role of surface imperfections at criticality, we consider the ferromagnetic nearest-neighbour Ising model on the simple cubic lattice for films of $L$ layers, using Monte Carlo and the density-matrix renormalization group techniques. To be specific, we study films with a straight line of magnetic adatoms or a straight step of monoatomic height. Both types of defects, lines and steps, are assumed to run across the entire surface layer through its center and along one of the crystallographic axes. The magnetic couplings at the surface, $J_{s}$, may be identical or different from those in the bulk, $J_{b}$. The local interactions at the defect may be modified, e.g., the couplings between neighbouring spins in the additional line [3].

Let us first discuss results for films with lines of magnetic adatoms (our work has been motivated by related experiments [5], albeit there the lines of adatoms are not coupled magnetically to the substrate).- The temperature, at which the transition between the ferromagnetic and the paramagnetic phases occurs, rises with the film thickness, $T_{c}=T_{c}(L)$, as shown in Fig.1. Of course, the magnetization in the additional line, $m_{l}$, is usually different from the surface magnetization, see also Fig. 1, because of the reduced coordination number and the, possibly, modified local couplings.

To elucidate critical properties at the line as compared to those of the perfect surface and of the bulk, one may monitor the effective exponent $\beta_{\text {eff }}$. That quantity follows from the ansatz for the magnetization $m \propto t^{\beta_{e f f}}$, where $t$ is the reduced temperature $\left|T_{c}(L)-T\right| / T_{c}(L)$. For $t \longrightarrow 0, \beta_{\text {eff }}$ becomes the true critical exponent $\beta$.- In accordance with general considerations on critical phenomena at defect lines in systems with two-dimensional critical fluctuations [6], we find the critical exponent $\beta_{l}$ of the magnetization in the line of adatoms to be non-universal. In films of finite thickness $L$, it varies from 0 to $1 / 2$, decreasing when strengthening the local couplings at the line, and depending only rather weakly on $L[3]$. In contrast, the critical exponent of the magnetization of a perfect surface is $1 / 8$ for all finite values of $L$. It remains to be $1 / 8$ even in the thermodynamic limit, $L \longrightarrow \infty$, when the surface orders at a higher temperature than the bulk ('surface transition') due to sufficiently strong interactions in the surface, $J_{s}$. When the surface and the bulk order at the same temperature ('ordinary transition'), the critical exponent of the surface magnetization is, in the limit $L \longrightarrow \infty$, about 0.80 ; this quite large value leads, for finite $L$, to a pronounced increase of the effective exponent away from criticality, both for the magnetization of the surface and in the line [3]. That behaviour should be taken into account in interpreting properly, e.g., 


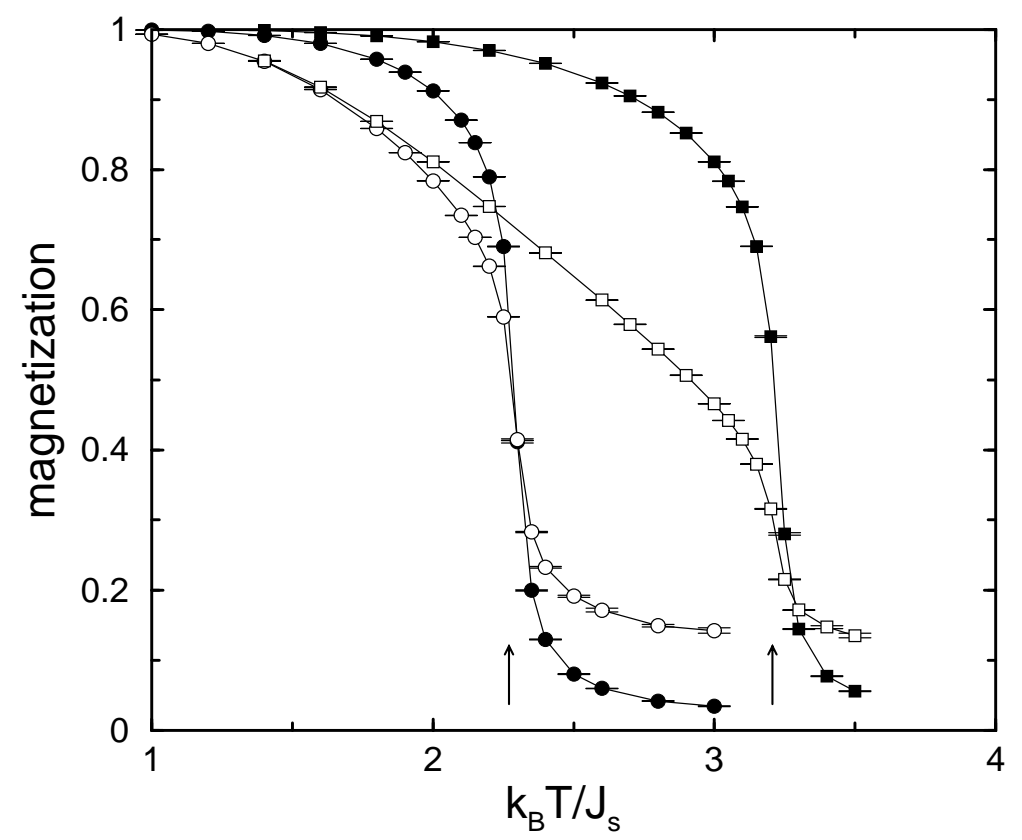

Fig. 1. Simulated magnetization in the line of adatoms (open symbols) and of the perfect surface (full symbols) of Ising models with equal couplings for $L=1$ (circles) and 2 (squares); each layer has $81 \times 80$ spins.

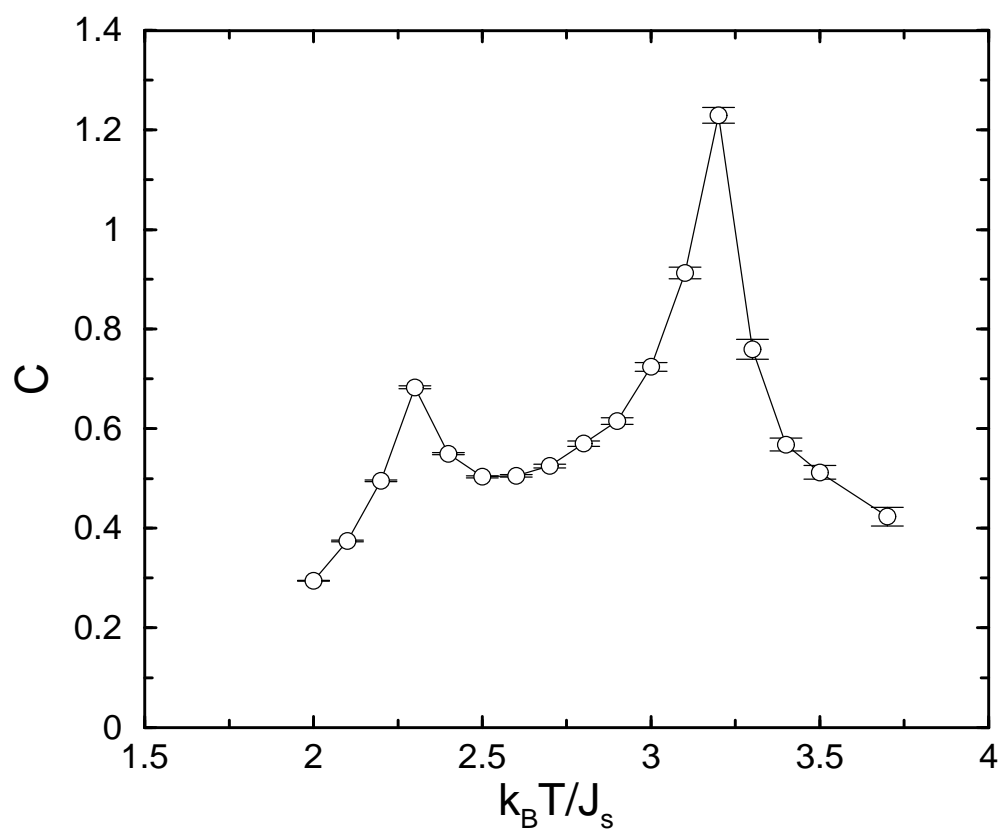

Fig. 2. Simulated specific heat $C$ of the Ising model with equal couplings and with a step dividing the system into two halfs of one and two layers; the bottom layer consists of $120 \times 120$ spins. 


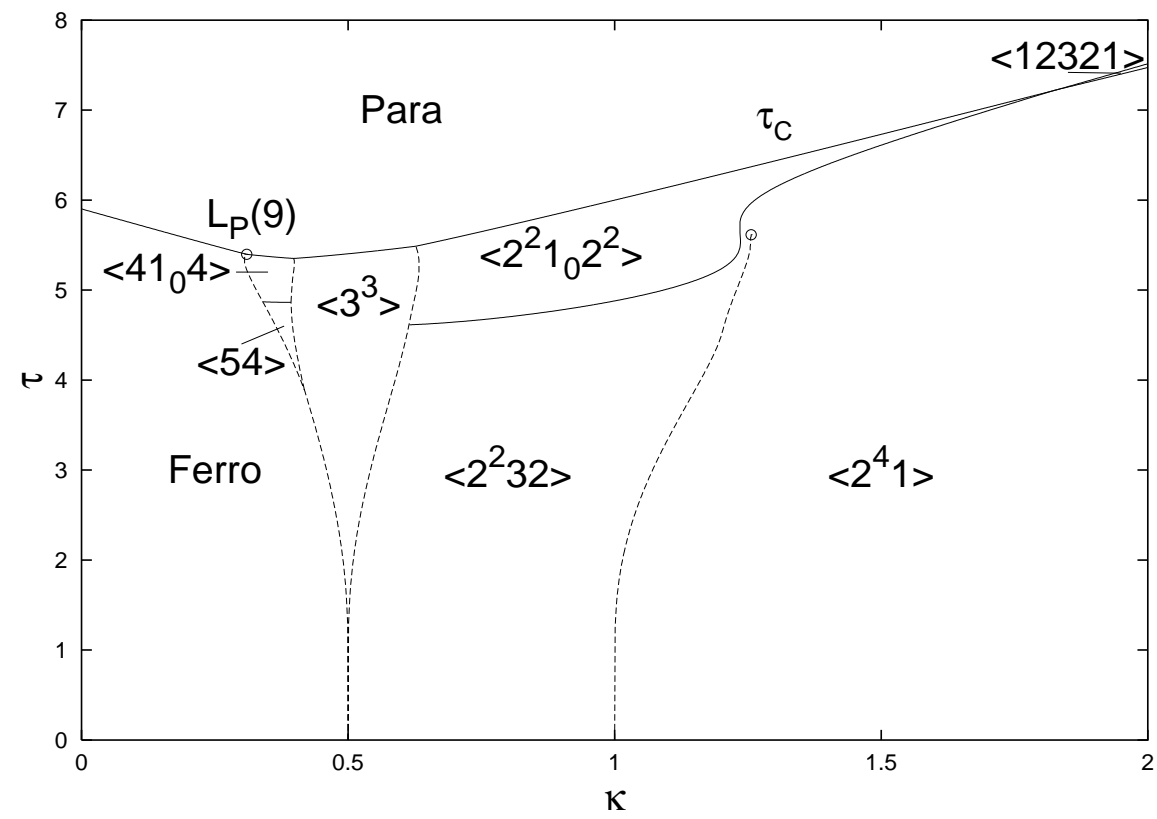

Fig. 3. Phase diagram of the ANNNI model with 9 layers, in mean-field approximation; $\tau=k_{B} T / J_{0}$. Phases are denoted in the usual manner, Refs. 4 and 8.

experimental data.

Another type of surface defect is the straight step of monoatomic height, dividing the film in two halfs of thickness $L$ and $L+1$. Accordingly, we are dealing with a composite system displaying two distinct phase transitions at $T_{c}(L)$ and $T_{c}(L+1)$ [3], as signalled, e.g., by the two-peak structure of the specific heat, see Fig. 2 (attention may be drawn to related recent experimental observations [7]). Because $T_{c}(L+1)>T_{c}(L)$, the magnetization at the step edge is expected to vanish on approach to $T_{c}(L+1)$ similarly to the magnetization at the surface of a two-dimensional Ising model. Thence, the critical exponent ist expected to be $1 / 2$ [6], in accordance with our simulational findings [3].

Secondly, we study the influence of the film thickness on spatially modulated magnetic phases in the framework of the ANNNI model [8] on the simple cubic lattice with $L$ layers [4]. Neighbouring spins in each of the $L$ layers are coupled ferromagnetically, $J_{0}>0$. Perpendicular to the layers ferromagnetic interactions, $J_{1}>0$, between neighbouring spins in adjacent layers, compete with antiferromagnetic couplings, $J_{2}<0$, between axial next-nearest neighbour spins. Our work extends a recent analysis on the influence of the film surface on critical properties of the Lifshitz point [9].

For each film thickness $L$, we determine, using mean-field theory, Monte Carlo simulations and low-temperature series, a distinct phase diagram (setting $\left.J_{0}=J_{1}\right)$, in the $\left(\kappa=-J_{2} / J_{1}, k_{B} T / J_{0}\right)$ plane, consisting of various modulated phases. The cases $L=5,7$, and 8 have been depicted before [4]. In Figs. 3 and 4 , we show the mean-field phase diagrams for $L=9$ and 10 . 


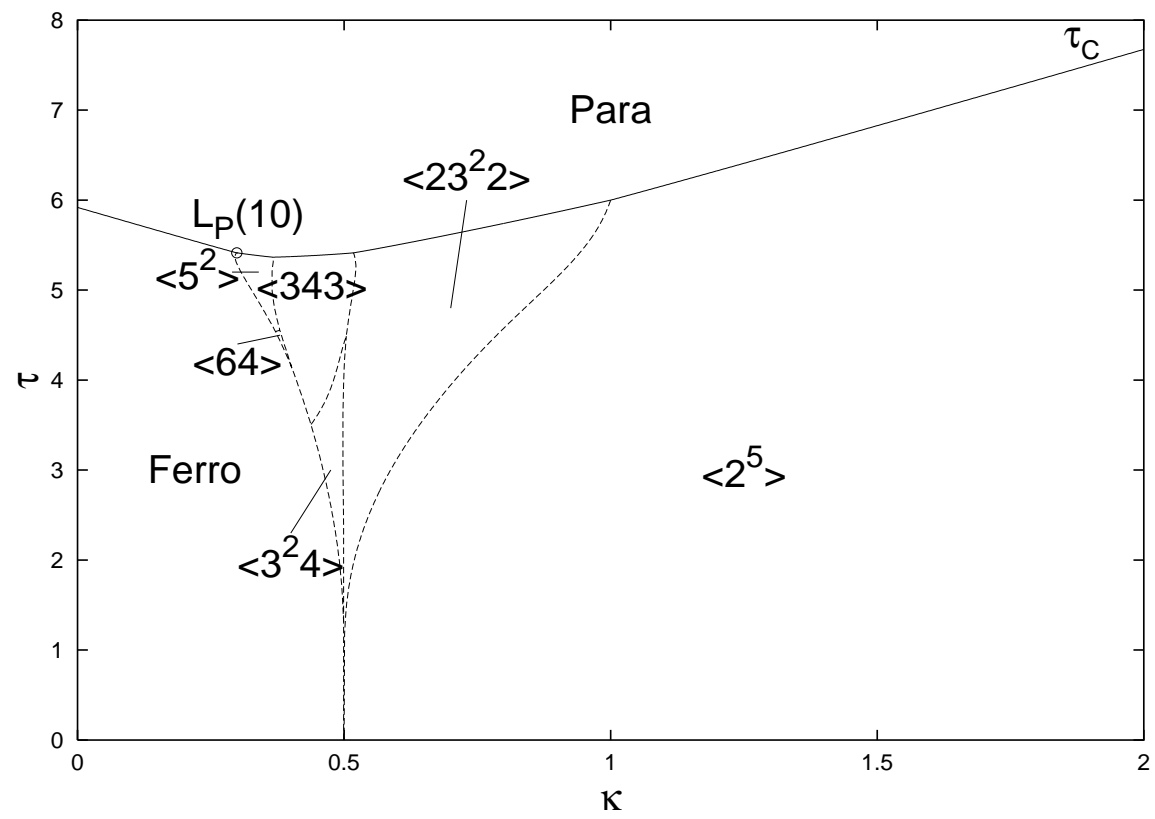

Fig. 4. Phase diagram of the ANNNI model with 10 layers, in mean-field approximation.

Compared to the ANNNI model in the thermodynamic limit, $L \longrightarrow \infty$, main differences due to the finite film thickness are: (i) The ordered phases occurring directly below the transition line to the paramagnetic phase are either symmetric or antisymmetric about the center of the film. In fact, neighbouring phases have different parity, with their number of extrema in the layer magnetization $m(z), z=1,2, \ldots L$, increasing by one when increasing $\kappa$. (ii) For $L$ odd, the center layer of the antisymmetric variants of those phases is disordered ('partially disordered phases'). (iii) For $L$ odd, a transition line arises from $(\kappa=1, T=0)$, in addition to the multiphase point at $(\kappa=1 / 2, T=0)$ being present at all $L$. The phases springing from the multiphase point may include structures (having one 4-band or one 5-band of layers with equally oriented spins) being not stable in the thermodynamic limit.

Of course, it is of interest to study the robustness of our findings against modifications of the model parameters. In any event, results may provide some guidance to explain possible experimental realizations for thin films with magnetic superstructures (here, attention is drawn to recent local-spin-density calculations of thin $\mathrm{Fe}$ films on $\mathrm{Co}$ substrates showing distinct and complex phase diagram for various film thicknesses [10]).

\section{References}

[1] U. Gradmann, J. Magn. Magn. Mater. 100, 481 (1991). 
[2] P. Poulopoulos and K. Baberschke, J. Phys.-Condens. Mat. 11, 9495 (1999).

[3] M.-C. Chung, M. Kaulke, I. Peschel, M. Pleimling, and W. Selke, Eur. Phys. J. B 18, 655 (2000).

[4] W. Selke, D. Catrein, and M. Pleimling, J. Phys. A 33, L459 (2000).

[5] J. Shen, R. Skomski, M. Klaua, H. Jenniches, S. Sundar Manoharan, and J. Kirschner, Phys. Rev. B 56, 2340 (1997)

[6] F. Iglói, I. Peschel, and L. Turban, Adv. Phys. 42, 683 (1993).

[7] S. Andrieu, C. Chatelain, M. Lemine, B. Berche, and Ph. Bauer, Phys. Rev. Lett. 86, 3883 (2001).

[8] M. E. Fisher and W. Selke, Phys. Rev. Lett. 44, 1502 (1980).

[9] K. Binder and H. L. Frisch, Eur. Phys. J. B 10, 71 (1999).

[10] D. Spisak and J. Hafner, Phys. Rev. B 62, 9575 (2000). 